\title{
Electromagnetic Transient Simulation of Large Power Transformer Internal Fault
}

\author{
Jun Liu ${ }^{1, ~ a ~, ~ S h i w u ~ X i a o ~}{ }^{1, b}$, Qingsen Sun ${ }^{2, c}$, Huaxing Wang ${ }^{2, d}$ and Lei Yang ${ }^{2, e}$ \\ ${ }^{1}$ School of Electrical \& Electronic Engineering, North China Electrical Power University, Changpin, \\ Beijing, China \\ ${ }^{2}$ State Grid Shandong Electric Power Company Tai'an Power Supply Company, Tai'an, Shandong, \\ China

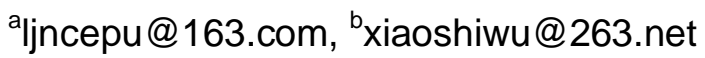

Keywords: parameters of transformer; internal fault; simulation; mathematical model.

Abstract. In this paper, in the neglect of the core of the magnetic hysteresis, eddy current effect and the core skin effect and based on the analysis and summary of previous experience, an analytical method to determine the parameters of the transformer's turn to turn short circuit fault is improved and perfected in order to determine the parameters of the transformer when the turn-to-turn fault and turn-to-ground fault happens at any position of the transformer. Through the mathematical model of transformer after fault, we can obtain the node voltage equation of the simulation network. And then we adopt three principles to calculate the unknown parameters in the node voltage equation we obtained before. Simulation results verify the effectiveness of the proposed algorithm.

\section{Introduction}

For transformer internal fault model, there are two methods for calculating the parameters: divide the coil into several units according to the structure of transformer coil and fault types, and calculate the inductance parameters between these unit coils directly; regard the inductance parameter of the normal operation of the transformer as the known quantity, and the inductance parameters of the fault transformer are determined by solving the equations. The first method requires to calculate the distribution of leakage magnetic field in transformer internal fault, it is very complex to calculate, so this paper uses the second method[1].

In this paper, due to the need for problem we study, the appropriate simplification can be adopted. In the neglect of the core of the magnetic hysteresis, eddy current effect and the core skin effect and based on the analysis and summary of previous experience, an analytical method to determine the parameters of the transformer's turn to turn short circuit fault is improved and perfected in order to determine the parameters of the transformer when the turn-to-turn fault and turn-to-ground fault happens at any position. Through the mathematical model of transformer after fault, we can obtain the node voltage equation of the simulation network. Simulation results verify the effectiveness of the proposed algorithm.

\section{Mathematical Model of Transformer Internal Fault Simulation}

The simulation model of the transformer must be able to simulate all the operating conditions for the algorithm, and the internal fault simulation of the transformer can be performed particularly. EMTP provides the self inductance and mutual inductance of the simulation algorithm and the branch number is not restricted, so we can regard the transformer as the self inductance and mutual inductance branch composed of the coils. So the mutual coupling branch model in the ATP can be used to simulate transformer. The normal transformer can be regarded as combination of several mutual magnetic coupling branches[2]. If the turn-to-turn fault or turn-to-ground fault in a certain coil of the transformer occurs, then coil in which the turn-to-turn fault occurs can be viewed as two coupling coils separately, and the transformer in which the turn-to-ground fault occurs can be considered as three mutual coupling independent coils. 
Take three-phase double-winding transformer as an example to describe the above principles in detail. Under normal circumstances the three-phase double-winding transformer can be regarded as six branches in which mutual inductance exists, as is shown in Fig.1. This way, a three-phase two-winding transformer can be expressed by the sixth-order branch resistance matrix [R] and the sixth-order branch inductance matrix [L], as is shown in equation (1).

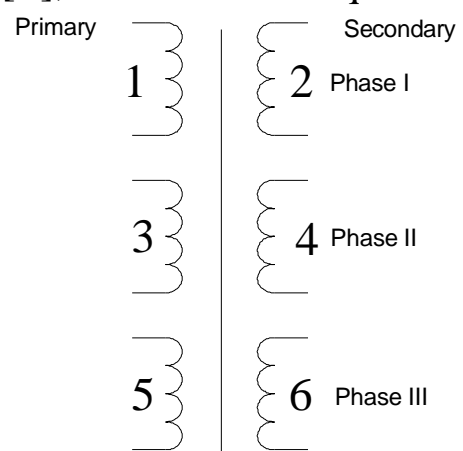

Fig.1. multi-phase coupled branch model of transformer

$$
[R]=\left[\begin{array}{cccccc}
R_{1} & 0 & 0 & 0 & 0 & 0 \\
0 & R_{2} & 0 & 0 & 0 & 0 \\
0 & 0 & R_{3} & 0 & 0 & 0 \\
0 & 0 & 0 & R_{4} & 0 & 0 \\
0 & 0 & 0 & 0 & R_{5} & 0 \\
0 & 0 & 0 & 0 & 0 & R_{6}
\end{array}\right]_{6 \times 6} \quad[L]=\left[\begin{array}{cccccc}
L_{1} & M_{12} & M_{13} & M_{14} & M_{15} & M_{16} \\
M_{21} & L_{2} & M_{23} & M_{24} & M_{25} & M_{26} \\
M_{31} & M_{32} & L_{3} & M_{34} & M_{35} & M_{36} \\
M_{41} & M_{42} & M_{43} & L_{4} & M_{45} & M_{46} \\
M_{51} & M_{52} & M_{53} & M_{54} & L_{5} & M_{56} \\
M_{61} & M_{62} & M_{63} & M_{64} & M_{65} & L_{6}
\end{array}\right]_{6 \times 6}
$$

Among them, $R_{i}$ is the resistance of each coil in Fig.1, and $L_{i}$ is the self-inductance of the coil, $M_{i j}$ is the mutual inductance between coil i and $\mathrm{j}(i=(1,2,3,4,5,6), j=(1,2,3,4,5,6), \quad i \neq j)$.

We divide the internal fault of transformer into two types: turn-to-turn fault and turn-to-ground fault, Fig.2 is a schematic diagram of three-phase double-winding transformer when a turn-to-ground fault occurs. It can be considered as seven branches in which mutual inductance exists, and it can be represented by seventh-order branch resistance matrix $[\mathrm{R}]$ and seventh-order branch inductance matrix [L], as is shown in equation(2).

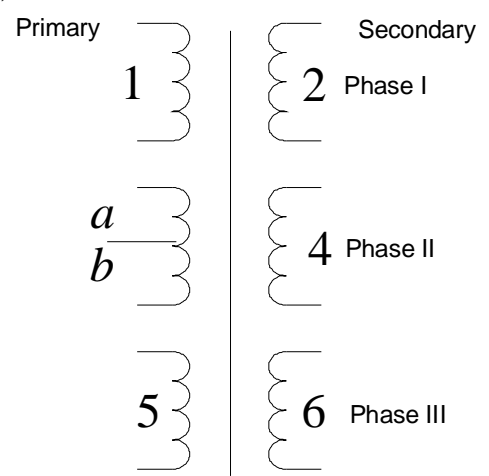

Fig.2. diagram of three-phase two-winding transformer when turn-to-ground fault occurs

$$
[R]=\left[\begin{array}{ccccccc}
R_{1} & 0 & 0 & 0 & 0 & 0 & 0 \\
0 & R_{2} & 0 & 0 & 0 & 0 & 0 \\
0 & 0 & R_{a} & 0 & 0 & 0 & 0 \\
0 & 0 & 0 & R_{b} & 0 & 0 & 0 \\
0 & 0 & 0 & 0 & R_{4} & 0 & 0 \\
0 & 0 & 0 & 0 & 0 & R_{5} & 0 \\
0 & 0 & 0 & 0 & 0 & 0 & R_{6}
\end{array}\right]_{7 \times 7} \quad[L]=\left[\begin{array}{ccccccc}
L_{1} & M_{12} & M_{1 a} & M_{1 b} & M_{14} & M_{15} & M_{16} \\
M_{21} & L_{2} & M_{2 a} & M_{2 b} & M_{24} & M_{25} & M_{26} \\
M_{a 1} & M_{a 2} & L_{a} & M_{a b} & M_{a 4} & M_{a 5} & M_{a 6} \\
M_{b 1} & M_{b 2} & M_{b a} & L_{b} & M_{b 4} & M_{b 5} & M_{b 6} \\
M_{41} & M_{42} & M_{4 a} & M_{4 b} & L_{4} & M_{45} & M_{46} \\
M_{51} & M_{52} & M_{5 a} & M_{5 b} & M_{54} & L_{5} & M_{56} \\
M_{61} & M_{62} & M_{6 a} & M_{6 b} & M_{64} & M_{65} & L_{6}
\end{array}\right]_{7 \times 7}
$$


Among them, $R_{i}$ is the resistance of each coil in Fig. $2, L_{i}$ is the self-inductance of each coil, $M_{i j}$ is the mutual inductance between the coil i and $\mathrm{j}(i=(1,2, a, b, 4,5,6), j=(1,2, a, b, 4,5,6), i \neq j)$.

Fig. 3 is a schematic diagram of the transformer when turn-to-turn fault occurs. Similarly, the transformer can be seen at this time as eight mutual inductance branches, and represented by eighth-order inductance matrix and eighth-order resistance matrix.

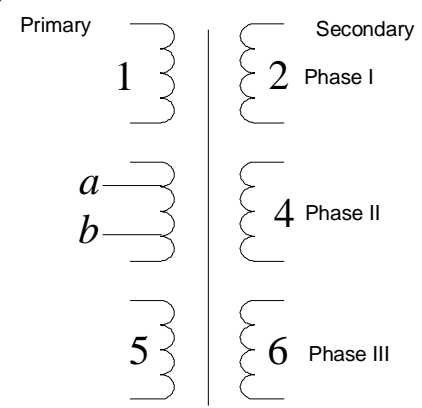

Fig.3. diagram of three-phase double-winding transformer when turn-to-turn fault occurs

The $8 * 8$ matrix [R] and [L] of the transformer when turn-to-turn fault occurs can also be obtained by using the same analytical method above.

The main purpose of this paper is to determine the parameters of corresponding $[R]$ and $[\mathrm{L}]$ in the seventh-order matrix or eighth-order matrix when turn-to-turn fault or turn-to-ground fault occurs by using the analytic method, and the use EMTP to simulate the current when turn-to-turn fault or turn-to-ground fault occurs.

\section{Short Circuit between Turns and Ground}

When the transformer turn-to-ground short circuit occurs, it is equivalent that a tap is extracted from a certain coil, as is shown in Fig.2. The parameters in matrix [R] and [L] of the transformer changes into seventh-order matrix respectively, as is shown in formula (2).

In view of the convenience of problem analysis and practical needs of the project, in the matrix (2), the parameters of the non-fault coil can be considered as the same before and after the fault [3]. For example $R_{1}, R_{2}, R_{4}, R_{5}$ and $R_{6}$, unknown parameters are $R_{a}$ and $R_{b}$, they can be expressed as

$$
R_{a}=\left(\frac{n_{a}}{n_{3}}\right) R_{3} \quad R_{b}=\left(\frac{n_{b}}{n_{3}}\right) R_{3}
$$

In matrix [L], Some parameters associated with the non-fault coil can be considered to be approximately constant, for example $L_{1}, L_{5}, M_{12}, M_{61}$ and so on. And some parameters related to the fault coil is changed before and after the fault, for example $L_{a}, L_{b}, M_{1 a}, M_{6 b}$ and so on. Therefore, we need to determine the changes of the parameters of the transformer after the fault.

For the calculation of the fault parameters of the transformer after fault, the following three principles are used in this paper.

(1) the principle of consistency

When using the $7 * 7$ matrix to analyze turn-to-ground fault, series connection of the coil a and the coil $\mathrm{b}$ is equivalent to $6 * 6$ matrix, as is shown in Fig.4.

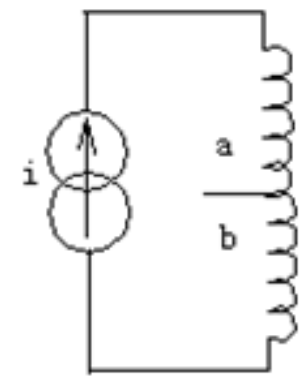

Fig.4. series equivalent circuit of fault coil when turn-to-ground fault occurs 
Assuming that the coil a and the coil $\mathrm{b}$ are connected in series and the current flowing in them is $\mathrm{i}$, and other coils have no load, according to Fig.4, flux equation can be listed as follows[4]:

$$
\left\{\begin{array}{l}
\Phi_{a}=\left(L_{a}+M_{a b}\right) i \\
\Phi_{b}=\left(L_{b}+M_{a b}\right) i
\end{array}\right.
$$

Before the fault, the magnetic flux in winding 3 is

$$
\Phi_{3}=L_{3} i
$$

Inductor formula when the two windings are connected in series can be drawn from the above two expressions, namely:

$$
L_{a}+2 M_{a b}+L_{b}=L_{3}
$$

Here, $\mathrm{L}_{\mathrm{a}}$ and $\mathrm{L}_{\mathrm{b}}$ are the elements of the $7 * 7$ order matrix represented by the formula (2).

(2) leakage coefficient

The transformer current when turn-to-ground fault occurs largely depends on the size of the magnetic flux leakage, therefore, the magnetic flux leakage factor between coil a and coil $b$ must be considered[5]. Magnetic flux leakage factor can be expressed as:

$$
\sigma_{a b}=1-\frac{M_{a b}^{2}}{L_{a} L_{b}}
$$

(3) proportionality

It can be approximated that self-inductor of wingdings is proportional to the square of number of turns. This can meet the requirements of accuracy in the case of a very small leakage coefficient.

$$
\frac{L_{a}}{L_{b}}=\left(\frac{n_{a}}{n_{b}}\right)^{2}
$$

Consider the above three equations. $\mathrm{k}$ represents where the location of the fault is said to occur. $\mathrm{n}_{\mathrm{a}}$ and $\mathrm{n}_{\mathrm{b}}$ can be used as the known quantity in the fault transient simulation software, therefore, we can use equation(6), (7) and (8) to obtain $L_{a}, L_{b}$ and $M_{a b}$, their expressions are:

$$
\begin{aligned}
& L_{a}=\frac{L_{3}}{\frac{1}{k^{2}}+\frac{2 \sqrt{1-\sigma_{a b}}}{k}+1} \\
& L_{b}=\frac{L_{3}}{k^{2}+2 k \sqrt{1-\sigma_{a b}}+1} \\
& M_{a b}=\frac{L_{3} \sqrt{1-\sigma_{a b}}}{\left(k+\frac{1}{k}\right)+2 \sqrt{1-\sigma_{a b}}}
\end{aligned}
$$

The method of solving the mutual inductance between coil a and the other coils is discussed below. Generally speaking, mutual induction before and after the fault complies with following equation:

$M_{3 i}=M_{a i}+M_{b i}$

In general, the mutual inductance of the winding is proportional to the number of turns[6], that is:

$$
\frac{M_{a i}}{M_{b i}}=\frac{n_{a}}{n_{b}}=k
$$


By the formula (12) and (13) we can obtain:

$$
\left\{\begin{array}{l}
M_{a i}=\frac{k}{1+k} M_{3 i} \\
M_{b i}=\frac{1}{1+k} M_{3 i}
\end{array}\right.
$$

By using the similar method, the parameters of $[\mathrm{L}]$ and $[\mathrm{R}]$ in the $8 * 8$ order matrix corresponding to the turn-to-turn short circuit can also be obtained.

\section{Internal Fault Simulation of Transformer}

In this paper, the correctness of the algorithm is verified by the example of the Dy11 connection of $t$ ransformer. The power supply parameter: amplitude of rated voltage is $120 \mathrm{~V}$, frequency is $50 \mathrm{~Hz}$, in ternal resistance is $0.002 \mathrm{ohm}$, reactance is $0.062 \mathrm{ohm}$; line parameter: resistance is $0.003 \mathrm{ohm}$, react ance is $0.094 \mathrm{ohm}$; The parameters of three-phase transformer are as follows: rated voltage $U=115 / 300 \pm 2 \times 2.5 \% \mathrm{~V}$; winding resistance $R=0.2 / 0.8 \Omega$; leakage reactance: $L=0.002 / 0.01 \mathrm{H}$.

In Fig.5, the fault occurs on the A phase winding of the high voltage coil, and the short circuit oc curs at the point of $20 \%$ of the winding. The A,B,C three-phase current, as shown in Fig.6, are obtai ned by using the above simulation method:

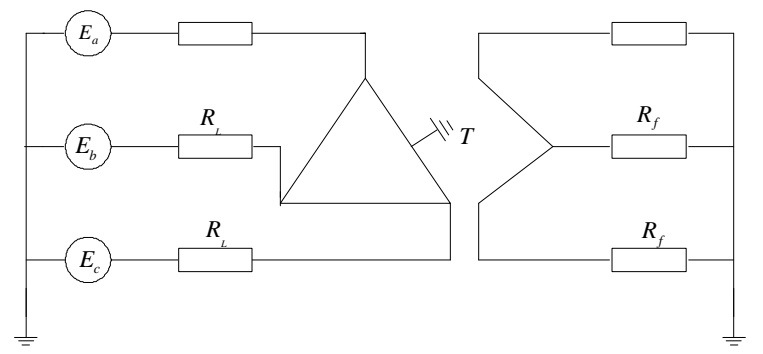

Fig.5. Diagram of transformer using Dy11 connection form when turn-to-ground fault occurs
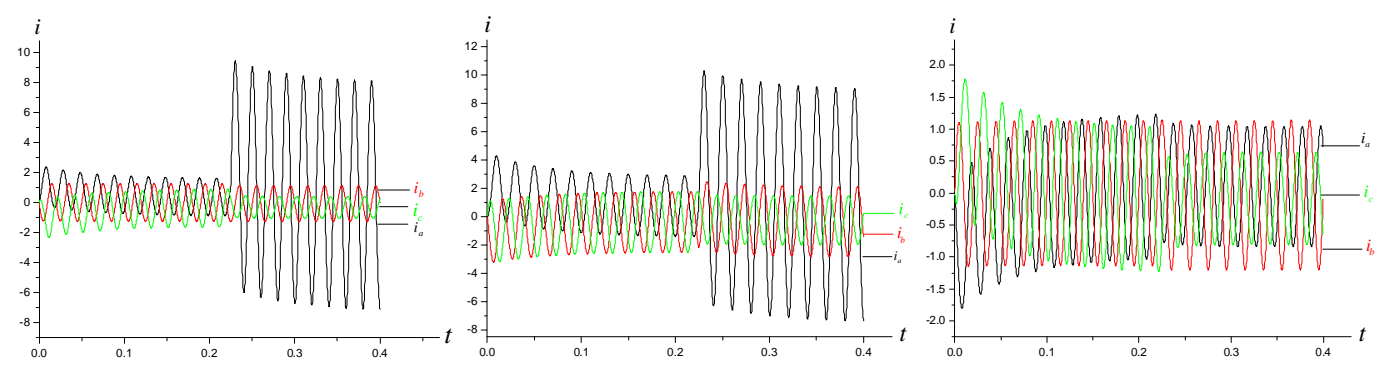

The primary phase currents The primary line current Secondary line current

Fig.6. Three phase current waveforms

In Fig.7, the fault occurs on the A phase winding of the high voltage coil. The winding turns ratio is 2:3:5 after the turn-to-turn short circuit. The A,B,C three-phase current, as shown in Fig.8, are obtained by using the above simulation method.
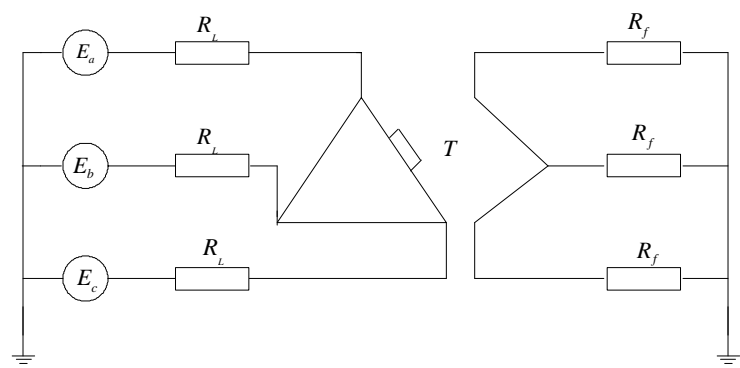

Fig.7. Schematic diagram of transformer using Dy11 connection form when turn-to-turn fault occurs 

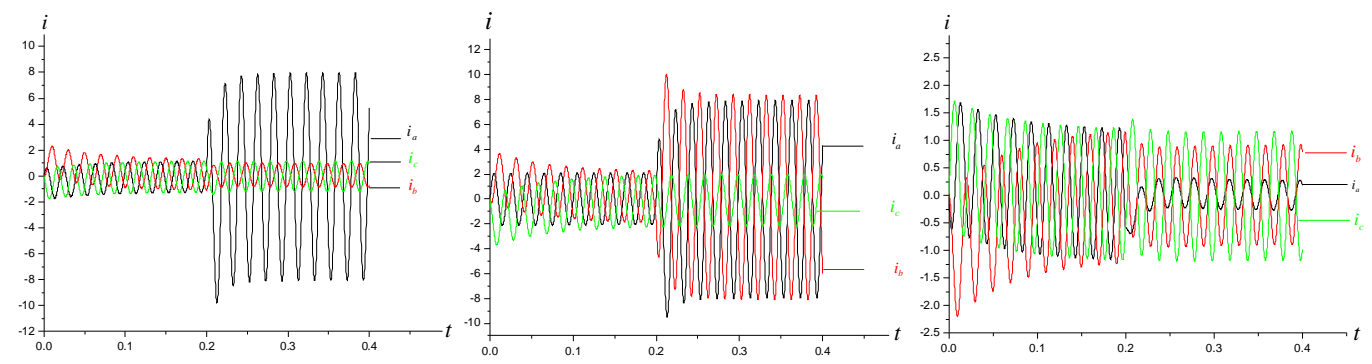

The primary phase currents The primary line current Secondary line current Fig.8 Three phase current waveforms

\section{Conclusion}

Due to the development of fault diagnosis and relay protection technology for electrical equipment, higher requirements for the understanding of the fault mechanism of the main electrical equipment are put forward. Especially for large transformer relay protection, only accurate analysis of the changes of the physical quantities in the transient and steady state of the transformer fault operation is carried out, and distribution and laws of the transformer current, voltage and other electrical quantity after fault is revealed, so that we can find out the measurable fault characteristic amount and use these characteristics or derived quantities to form a practical criterion for relay protection to achieve the improvement of the existing protection scheme or to propose new protection principle and scheme. In this paper, due to the need for problem we study, the appropriate simplification can be adopted. In the neglect of the core of the magnetic hysteresis, eddy current effect and the core skin effect and based on the analysis and summary of previous experience, an analytical method to determine the parameters of the transformer's turn-to-turn short circuit fault is improved and perfected. Simulation results verify the effectiveness of the proposed algorithm.

\section{References}

[1] Yi Zhu, Dazhong Fang, Power System Technology, J. 36 (2012) 116-120.

[2] Baohui Zhang, Xianggen Yin, Power System Protective Relaying, second ed., Beijing, 2010.

[3] Richang Qi, Power Transformer, first ed., Beijing, 2006.

[4] Xue Wang, Zengping Wang, Study of simulation of transformer with internal faults, J. Power System Technology. 28 (2004) 50-52 (in Chinese).

[5] Yongrong Huang, Qunzhan Li, Simulation for magnetic inrush and fault current of three phase transformer based on EMTDC, J. Relay. 35 (2007) 26-30 (in Chinese).

[6] Zeyi Zhao, Zhibizo Feng, Digital real time simulation model and digital integral of autotransformer, J. Journal of Tongji University. 29 (2001) 416-420 (in Chinese). 